NKU-SF2-2011

\title{
New Charged Dilaton Solutions in $2+1$ Dimensions and Solutions with Cylindrical Symmetry in 3+1 Dimensions
}

\author{
Sharmanthie Fernando ${ }^{1}$ \\ Department of Physics \& Geology \\ Northern Kentucky University \\ Highland Heights \\ Kentucky 41099 \\ U.S.A.
}

\begin{abstract}
We report a new family of solutions to Einstein-Maxwell-dilaton gravity in $2+1$ dimensions and Einstein-Maxwell gravity with cylindrical symmetry in $3+1$ dimensions. A set of static charged solutions in $2+1$ dimensions are obtained by a compactification of charged solutions in $3+1$ dimensions with cylindrical symmetry. These solutions contain naked singularities for certain values of the parameters considered. New rotating charged solutions in $2+1$ dimensions and $3+1$ dimensions are generated treating the static charged solutions as seed metrics and performing $S L(2 ; R)$ transformations.
\end{abstract}

Key words: Dilaton, Cylindrical, 2+1 Dimensions, Maxwell's.

\footnotetext{
${ }^{1}$ fernando@nku.edu
} 


\section{Introduction}

Three dimensional gravity has provided us with many important clues about higher dimensional physics. String theory, which seems to be the best candidate available for a consistent theory of quantum gravity, requires studies of low dimensional effective string actions. In this respect, dilaton gravity in $2+1$ dimensions deserve further attention since it arises from a low energy string effective theory.

In one of the earlier works, Shiraishi[1] found a family of static multi centered solutions for Einstein-Maxwell-dilaton gravity. Park and Kim [2] constructed general static axially symmetric solutions to the same model by dimensional reducing to two dimensions. In recent times, lot of attention has been given to gravity in $2+1$ dimensions with a negative cosmological constant due to the existence of black hole solutions [3]. Modification of this black hole with a dilaton and Maxwell's fields have lead to many interesting results. Static charged black holes and spinning black holes in anti-de Sitter space by Chan and Mann[4][5], spinning solutions with self dual electromagnetic fields by Fernando[6], black holes in a generalized dilaton gravity with a Brans-Dicke type parameter by Sá et.al.[7], magnetic solutions by Koikawa et.al.[8] are some of the work related to dilaton gravity in anti-de Sitter space.

In this paper we present an interesting class of dilaton solutions arising from four dimensional gravity. This is achieved by a compactification of electrically and magnetically charged solutions with cylindrical symmetry in four dimensions. These new set of solutions are different from the ones presented above due its direct relation to four dimensions. These solutions are important since it provide us with a clear understanding of how 4D gravity and 3D gravity are related to each other. Furthermore, we have generated rotating charged solutions to Einstein-Maxwell-dilaton gravity via "T-duality" and treating the compactified solutions as the seed metrics. Also, embedding these $2+1$ dimensional solutions in $3+1$ dimensions leads to new rotating charged solutions with cylindrical symmetry. Such a compactification from an uncharged cylindrical solution to $2+1$ dimensions is reported in Fernando [9].

We have structured the paper as follows. In section 2 we will give a brief introduction to four dimensional charged solutions with cylindrical symmetry and compactify them to obtain dilaton solutions in three dimensions. In section 3 and 4 we will study static charged solutions. In section 5 , new rotating charged solutions in $2+1$ dimensions are generated. In section 6 , new rotating charged solutions in $3+1$ dimensions with cylindrical symmetry are presented. Finally we will conclude.

\section{Compactification of Charged Solutions in Four Dimensions}

Cylindrical symmetrical space-times in four dimensions consists of isometries generated by two commuting space-like Killing vectors. If the solutions are stationary, then 
the space-time admit another Killing vector along the time axis. General stationary cylindrical symmetric line element with three Killing vectors $\partial_{t}, \partial_{z}$, and $\partial_{\varphi}$ can be written as,

$$
d s^{2}=e^{-2 U}\left(e^{2 K}\left(d r^{2}+d z^{2}\right)+r^{2} d \varphi^{2}\right)-e^{2 U}(d t+A d \varphi)^{2}
$$

where $U, K$ and $A$ are functions of $r$ only. The above metric is a solution to the Einstein-Maxwell action in four dimensions given by,

$$
S=\int d^{4} x \sqrt{-G}\left(R-F_{\alpha \beta} F^{\alpha \beta}\right)
$$

The purpose of this section is to dimensionally reduce four dimensional Einstein gravity to three dimensions to obtain dilaton gravity. If we pick the cylindrical solutions given above and treat the $z$ coordinate to be compact for the purposes of compactification, the reduction will yield a theory in three dimensions with gravity, dilaton field $\phi$ and the gauge field $A_{\mu}$.

Now, to perform compactification along the $z$ direction, let us rewrite the above metric in four dimensions as follows:

$$
d s_{3+1}^{2}=G_{\mu \nu} d x^{\mu} d x^{\nu}=g_{a b} d x^{a} d x^{b}+e^{-4 \phi} d z^{2}
$$

with

$$
e^{-4 \phi}=G_{z z}
$$

Here $(\mu, \nu=0,1,2,3)$ and $(a, b=0,1,2)$ are four and three dimensional indices respectively. The dimensionally reduced action in three dimensions is given by,

$$
S_{\text {string }}=\int d^{3} x \sqrt{-g^{S}} e^{-2 \phi}\left(R^{S}-F_{\alpha \beta} F^{\alpha \beta}\right)
$$

Here, $g_{a b}^{S}$ corresponds to $g_{a b}$ of the four dimensional metric and the metric can be treated to be in the "string frame". One can perform a conformal transformation to bring the metric to Einstein frame as follows,

$$
e^{-4 \phi} g_{\mu \nu}^{S}=g_{\mu \nu}^{E}
$$

This transformation will lead to the following action and the corresponding field equations,

$$
\begin{gathered}
S_{\text {Einstein }}=\int d^{3} x \sqrt{-g}\left(R-8 \nabla_{\mu} \phi \nabla^{\mu} \phi-e^{-4 \phi} F^{2}\right) \\
R_{\mu \nu}=8 \nabla_{\mu} \phi \nabla_{\nu} \phi+e^{-4 \phi}\left(-g_{\mu \nu} F^{2}+2 F_{\mu}^{\theta} F_{\nu \theta}\right) \\
8 \nabla_{\mu} \nabla^{\mu} \phi+2 e^{-4 \phi} F^{2}=0 \\
\nabla_{\mu}\left(e^{-4 \phi} F^{\mu \nu}\right)=0
\end{gathered}
$$




\section{$3 \quad$ Static Electrically Charged Dilaton Solutions}

First we will consider the static cylindrical solutions of Einstein-Maxwell gravity corresponding to a radial electric field caused by an axial charge distribution along the $\mathrm{z}$-axis is given as follows:

$$
\left.d s^{2}=r^{2 m^{2}} L^{2}\left(d r^{2}+d z^{2}\right)+r^{2} L^{2} d \varphi^{2}\right)-L^{-2} d t^{2}
$$

The function $L$ is given by,

$$
L=c_{1} r^{m}+c_{2} r^{-m}
$$

with $c_{1}, c_{2}$ and $m$ being real constants. This metric is a solution of the action in eq.(2). The electric field is given by

$$
F_{r t}=\frac{-Q}{L^{2} r}
$$

with,

$$
-4 c_{1} c_{2} m^{2}=Q^{2}
$$

Notice that $c_{1}$ and $c_{2}$ need to have opposite signs for the charge $Q$ to be real. In the following discussion we will consider $c_{1}<0$ and $c_{2}>0$. For $c_{1}=0$ and $c_{2}=1$, the above metric corresponds to Levi-Civita vacuum static solution[10]. The Mukherji [11] solution describing the gravitational field of a charged line-mass is contained in eq.(11). Now, by starting from the metric in eq.(11), one can obtain solutions to three dimensional space-time after compactification as,

$$
\left.d s_{\text {Ein }}^{2}=r^{4 m^{2}} L^{4} d r^{2}+r^{2+2 m^{2}} L^{4} d \varphi^{2}\right)-r^{2 m^{2}} d t^{2}
$$

The space-time in $2+1$ dimensions consists of a dilaton field given by,

$$
\phi=-\frac{1}{2} \ln \left(r^{m^{2}}\left(c_{1} r^{m}+c_{2} r^{-m}\right)\right)
$$

and a electric potential given by,

$$
A_{t}=\frac{Q}{2 c_{1} m} \frac{r^{-m}}{\left(c_{2} r^{-m}+c_{1} r^{m}\right)}
$$

The Ricci Scalar $R s=R_{\mu \nu} g^{\mu \nu}$, Kretschmann scalar $K=R_{\mu \nu \gamma \beta} R^{\mu \nu \gamma \beta}$ and $F^{2}=$ $F_{\mu \nu} F^{\mu \nu}$ are computed for the above metric as follows:

$$
R s=\frac{\left(2 m^{2} r^{-2+4 m-4 m^{2}}\right)}{\left(1+c_{1} r^{2 m}\right)^{6}}\left\{\left(1-2 m+m^{2}\right)+2 r^{2 m} c_{1}\left(m^{2}-3\right)+r^{4 m} c_{1}^{2}\left(1+2 m+m^{2}\right)\right\}
$$




$$
\begin{gathered}
K=\frac{\left(4 m^{4} r^{-4+8 m-8 m^{2}}\right)}{\left(1+c_{1} r^{2 m}\right)^{1} 2}\left\{3-12 m+18 m^{2}-12 m^{3}+3 m^{4}+\right. \\
r^{2 m}\left(-4 c_{1}+8 c_{1} m+8 c_{1} m^{2}-24 c_{1} m^{3}+12 c_{1} m^{4}\right)+r^{4 m}\left(50 c_{1}^{2}-20 c_{1}^{2} m^{2}+18 c_{1}^{2} m^{4}\right) \\
r^{4 m}\left(50 c_{1}^{2}-20 c_{1}^{2} m^{2}+18 c_{1}^{2} m^{4}\right)+r^{6 m}\left(-4 c_{1}^{3}-8 c_{1}^{3} m+8 c_{1}^{3} m^{2} 2\right) \\
\left.r^{8 m}\left(3 c_{1}^{4}+12 c_{1}^{4} m+18 m^{2} c_{1}^{4}+12 c_{1}^{4} m^{3}+3 c_{1}^{4} m^{4}\right)\right\} \\
F^{2}=\frac{-2 Q^{2}}{\left(c_{2}+c_{1} r^{2 m}\right)^{8}} r^{\left(-2+8 m-6 m^{2}\right)}
\end{gathered}
$$

\subsection{Case 1: $c_{1}=0$}

In this case the metric becomes,

$$
d s^{2}=r^{4 m^{2}-4 m} c_{2}^{4} d r^{2}+r^{2+2 m^{2}-4 m} c_{2}^{4} d \varphi^{2}-r^{2 m^{2}} d t^{2}
$$

and the charge $Q=0$. For $m=0$ the above metric can be shown to be flat when the coefficient $c_{2}$ is absorbed into the definition of $r$ and $t$. For $m=1$, the resulting metric can be shown to be flat by a coordinate transformation $\varphi \rightarrow i t$ and $t \rightarrow-i \varphi$. In fact one can observe that the scalar curvature $R s=0$ for $m=0,1$ and the dilaton,

$$
\phi=-\frac{1}{2}\left(m^{2}-m\right) \ln (r)
$$

is zero for $m=0,1$ as expected. But for $m \neq 0,1$ there will be a curvature singularity at $r=0$ (given $m \geq 0$ ) and the dilaton will be non-zero.

\subsection{Case 2: $c_{1} \neq 0$ and $c_{2}=1$}

When $c_{1} \neq 0$ and $m=0,1$, the charge $Q=0$ and the dilaton $\phi=0$ leading to a flat space-time. For $c_{1} \neq 0$ and $m \neq 0,1$, the electric charge is non-zero. Furthermore there are two curvature singularities in the metric, one at $r_{s 1}=\left(-1 / c_{1}\right)^{1 / 2 m}$ and the other at $r_{s 2}=0$. However, since $c_{1}<0$, the singularity at $r_{s 1}>0$ results in a naked singularity.

\section{Static Magnetically Charged Dilaton Solutions}

Here, we will consider static cylindrical symmetric solutions of Einstein-Maxwell gravity corresponding to a magnetic field along the z-axis. Physically it could correspond to the field interior to a solenoid current whose axis is the z-axis[10]. The metric for such a space-time is given by,

$$
\left.d s^{2}=r^{2 m^{2}} L^{2}\left(d r^{2}-d t^{2}\right)+L^{-2} d \varphi^{2}\right)+r^{2} L^{2} d z^{2}
$$


The function $L$ is given by,

$$
L=c_{1} r^{m}+c_{2} r^{-m}
$$

with $c_{1}, c_{2}$ and $m$ being real constants. The magnetic field is given by

$$
F_{r \varphi}=\frac{Q}{L^{2} r}
$$

with,

$$
4 c_{1} c_{2} m^{2}=Q^{2}
$$

Note that for the charge $Q$ to be real, both $c_{1}$ and $c_{2}$ should have the same sign in contrast to the electric case. For $m=1, c_{1}=-\frac{B_{0}^{2}}{4}$ and $c_{2}=-1$, the above metric corresponds to,

$$
d s^{2}=\left(1+\frac{B_{0}^{2} r^{2}}{4}\right)^{2}\left(d r^{2}+d z^{2}-d t^{2}\right)+\left(1+\frac{B_{0}^{2} r^{2}}{4}\right)^{-2} d \varphi^{2}
$$

This is widely known as Melvin magnetic universe (or "flux tube") [12] which represents a gravitational field generated by a uniform magnetic field $B_{0}$ along the z-axis.

One can obtain magnetically charged solutions to $2+1$ dimensional EinsteinMaxwell-dilaton gravity by compactifying the above metric in eq.(23) as follows:

$$
d s_{\text {Ein }}^{2}=r^{2+2 m^{2}} L^{4} d r^{2}+r^{2} d \varphi^{2}-r^{2+2 m^{2}} L^{4} d t^{2}
$$

with a dilaton,

$$
\phi=-\frac{1}{2} \ln \left(r\left(c_{1} r^{m}+c_{2} r^{-m}\right)\right)
$$

and a magnetic potential given by,

$$
A_{\varphi}=\frac{-Q}{2 c_{1} m} \frac{r^{-m}}{\left(c_{2} r^{-m}+c_{1} r^{m}\right)}
$$

The Ricci scalar $R s$, Kretschmann scalar $K$ and $F^{2}=F_{\mu \nu} F^{\mu \nu}$ are computed for the above metric as follows:

$$
\begin{gathered}
R s=\frac{r^{\left(-4+4 m-2 m^{2}\right)}}{\left(c_{2}+c_{1} r^{2 m}\right)^{6}}\left[c_{2}^{2}(1-m)^{2}+2 c_{1} c_{2} r^{2 m}\left(1-3 m^{2}\right)+c_{1}^{2} r^{4 m}(1+m)^{2}\right] \\
K=\frac{4 r^{-8+8 m-4 m^{2}}}{\left(c_{2}+c_{1} r^{2 m}\right)^{2}}\left\{3 c_{2}^{4}(1-m)^{4}+4 r^{2 m} c_{1} c_{2}^{3}\left(3-6 m+2 m^{2}+2 m^{3}-m^{4}\right)\right. \\
+r^{4 m}\left(c_{1} c_{2}\right)^{2}\left(18-20 m^{2}+50 m^{4}\right)+4 r^{6 m} c_{1}^{3} c_{2}\left(3+6 m+2 m^{2}-2 m^{3}-m^{4}\right) \\
\left.+3 r^{8 m} c_{1}^{4}(1+m)^{4}\right\} \\
F^{2}=\frac{2 Q^{2} r^{-6-2 m^{2}}}{\left(c_{2} r^{-m}+c_{1} r^{m}\right)^{8}}
\end{gathered}
$$




\subsection{Case 1: $c_{1}=0$}

In this case the metric becomes,

$$
d s^{2}=r^{4 m^{2}-4 m} c_{2}^{4} d r^{2}+r^{2+2 m^{2}-4 m} c_{2}^{4} d \varphi^{2}-r^{2 m^{2}} d t^{2}
$$

and the charge $Q=0$. Now, we will consider special values of $m$ to understand the structure of these space-times. First, for $m=0$ the above metric is,

$$
d s^{2}=r^{2} c_{2}^{4} d r^{2}+r^{2} d \varphi^{2}-r^{2} c_{2}^{4} d t^{2}
$$

and the dilaton $\phi=\frac{-1}{2} \ln \left[r c_{2}\right]$. From eq. $(31,32)$, one can observe that the scalar curvatures are non-zero for $m=0$. Hence in contrast to the electric case, the spacetime is not flat for $m=0$. For $m=1$, the metric is given by,

$$
d s^{2}=c_{2}^{4} d r^{2}+r^{2} d \varphi^{2}-c_{2}^{4} d t^{2}
$$

By redefining $r$ to be $r / c_{2}^{2}$ and $t$ to be $t c_{2}^{2}$, the above metric becomes,

$$
d s^{2}=d r^{2}+r^{2} c_{2}^{-4} d \varphi^{2}-d t^{2}
$$

and the dilaton $\phi=\frac{-1}{2} \ln \left[c_{2}\right]$. The scalar curvatures $R s$ and $K$ are zero. Hence, the space-time in eq.(37) corresponds to a conical singularity similar to the one obtained by Deser et.al.[13] and Gott[14]. The appearance of the conical singularity can be understood as follows: If we replace $\varphi$ with $\hat{\varphi}=\varphi / c_{2}^{2}$, then the metric eq.(37) corresponds to flat space-time locally. However, the former periodic coordinate $\varphi$ has the range $0 \leq \varphi \leq 2 \pi$ and the new period coordinate has the range $0 \leq \hat{\varphi} \leq 2 \pi c_{2}^{-2}$. Hence there is a deficit angle $D$ at the origin due to the presence of a massive source given by $D=2 \pi\left(1-c_{2}^{-2}\right)$ leading to the conical space-time. If $c_{2}=1$, then $D=0$ and the singularity vanishes. This is expected since the dilaton vanishes for $c_{2}=1$ leading to a vacuum. For $m \neq 0,1$, there is a curvature singularity at $r=0$ and is not covered by a horizon.

\subsection{Case 2: $c_{1} \neq 0$ and $c_{2}=1$}

In this case, if $m=0$ the electric charge is zero. However, in contrast to the electric case, the dilaton $\phi=-\frac{1}{2} \ln \left[r\left(c_{1}+c_{2}\right)\right]$ is not a constant. Observing the metric,

$$
d s^{2}=r^{2}\left(c_{1}+c_{2}\right)^{4}\left(-d t^{2}+d r^{2}\right)+r^{2} d \varphi^{2}
$$

it is clear that the space-time is not flat. Furthermore, there is a curvature singularity at $r=0$. For $m \neq 0$ the space-time is non-trivial and corresponds to a chargeddilatonic space-time. 


\section{Generating Rotating Charged Solutions in $2+1$ Dimensions}

Treating the above static charged solutions as seed metrics, one can generate rotating charged solutions in $2+1$ dimensions. Here, we adopt a solution generating technique developed by Chan[15]. Note that the static charged solutions presented in the previous section have two commuting Killing vectors $\partial_{t}$ and $\partial_{\varphi}$. One can write a general metric with such isometries as,

$$
d s^{2}=h_{i j} d x^{i} d x^{j}+e^{\chi} P(r)^{-2} d r^{2}
$$

where

$$
x^{i}=(\varphi, t) ; \quad \chi=\ln |\operatorname{det} h| ; \quad \text { and } \quad A=A_{i} d x^{i}
$$

Here, $A_{r}$ is choosen to be zero as a pure gauge quantity. Now, substituting the metric in eq.(39) in action eq.(7), and dividing it by the two-dimensional volume of the Killing orbits, one arrives at,

$$
S=\int d r\left\{P\left(\frac{\chi^{\prime 2}}{8}+\frac{1}{4} \operatorname{Tr}\left(H^{\prime} H^{\prime-1}\right)-8 \phi^{\prime 2}-2 e^{-4 \phi-\chi / 2} \operatorname{Tr}\left(A^{\prime T} H^{-1} A^{\prime}\right)\right)\right\}
$$

Here,

$$
H_{i j}=e^{-\chi / 2} h_{i j} ; \quad H_{i j}^{-1}=e^{\chi / 2} h^{i j} \quad \text { and } A \text { is a column vector of } A_{i} .
$$

The above action is invariant under $S L(2 ; R)$ transformations given by,

$$
H \rightarrow \Omega^{T} H \Omega, \quad A \rightarrow \Omega^{T} A \quad \text { where } \Omega \in S L(2 ; R)
$$

The above symmetry in the Lagrangian is similar to the "target space" symmetry arising in classical string effective action given by the "T-duality" group $O(1,2 ; R)$ [16]. Now, to perform the transformations in eq.(43), an arbitrary value of $\Omega$ can be parameterized as,

$$
\Omega=e^{\gamma J_{+}} e^{\eta J_{1}} e^{\beta J_{-}}
$$

Here, $J_{a}$ are $S L(2 ; R)$ generators which has matrix representation,

$$
J_{0}=\left(\begin{array}{cc}
0 & 1 \\
-1 & 0
\end{array}\right) \quad ; J_{1}=\left(\begin{array}{cc}
-1 & 0 \\
0 & 1
\end{array}\right) \quad ; J_{2}=\left(\begin{array}{cc}
0 & 1 \\
1 & 0
\end{array}\right)
$$

and $J_{ \pm}=\left(J_{2} \pm J_{0}\right) / 2$. Taking $e^{-\eta}=\alpha$ in the parameterization in eq.(44) will lead to the following $\Omega$ :

$$
\Omega=\left(\begin{array}{cc}
\alpha & \alpha \beta \\
\beta \gamma & \alpha^{-1}+\alpha \beta \gamma
\end{array}\right)
$$




\subsection{Rotating Electrically Charged Dilaton Solutions}

Considering the static charged metric in eq.(15) as the seed metric, one can apply the transformations given above to obtain the new metric as,

$$
d s_{\text {new }}^{2}=\tilde{h}_{i j} d x^{i} d x^{j}+r^{4 m^{2}} L^{4} d r^{2}
$$

where $\tilde{h_{i j}}$ are given by,

$$
\begin{gathered}
\tilde{h}_{t t}=-r^{2 m^{2}} \alpha^{2}+r^{2+2 m^{2}}\left(c_{2} r^{-m}+c_{1} r^{m}\right)^{4} \alpha^{2} \gamma^{2} \\
\tilde{h}_{t \varphi}=-r^{2 m^{2}} \alpha^{2} \beta+r^{2+2 m^{2}}\left(c_{2} r^{-m}+c_{1} r^{m}\right)^{4} \alpha \gamma\left(\alpha^{-1}+\alpha \beta \gamma\right) \\
\tilde{h}_{\varphi \varphi}=-r^{2 m^{2}} \alpha^{2} \beta^{2}+r^{2+2 m^{2}}\left(c_{2} r^{-m}+c_{1} r^{m}\right)^{4}\left(\alpha^{-1}+\alpha \beta \gamma\right)
\end{gathered}
$$

The new potential is,

$$
\tilde{A}_{i} d x^{i}=\frac{\alpha Q}{2 c_{1} m} \frac{r^{-m}}{\left(c_{2} r^{-m}+c_{1} r^{m}\right)}(d t+\beta d \varphi)
$$

The dilaton remains the same under these transformations.

\subsection{Rotating Magnetically Charged Dilaton Solutions}

Considering the magnetically charged static metric in eq.(28) as the seed metric, one can apply the transformations given above to obtain the new metric as,

$$
d s_{\text {new }}^{2}=\tilde{h}_{i j} d x^{i} d x^{j}+r^{2+2 m^{2}} L^{4} d r^{2}
$$

where $\tilde{h_{i j}}$ are given by,

$$
\begin{gathered}
\tilde{h}_{t t}=r^{2} \alpha^{2} \gamma^{2}-r^{2+2 m^{2}}\left(c_{2} r^{-m}+c_{1} r^{m}\right)^{4} \alpha^{2} \\
\tilde{h}_{t \varphi}=r^{2} \alpha \gamma\left(\alpha^{-1}+\alpha \beta \gamma\right)-r^{2+2 m^{2}}\left(c_{2} r^{-m}+c_{1} r^{m}\right)^{4} \alpha^{2} \beta \\
\tilde{h}_{\varphi \varphi}=r^{2}\left(\alpha^{-1}+\alpha \beta \gamma\right)^{2}-r^{2+2 m^{2}}\left(c_{2} r^{-m}+c_{1} r^{m}\right)^{4} \alpha^{2} \beta^{2}
\end{gathered}
$$

The new potential is,

$$
\tilde{A}_{i} d x^{i}=\frac{-\alpha \gamma Q}{2 c_{1} m} \frac{r^{-m}}{\left(c_{2} r^{-m}+c_{1} r^{m}\right)}\left(d t+\left(\alpha^{-2} \gamma^{-1}+\beta\right) d \varphi\right)
$$

The dilaton remains the same under these transformations. 


\section{Rotating Charged Solutions in 3+1 Dimensions with Cylindrical Symmetry}

Solutions with cylindrical symmetry in $3+1$ dimensions have interesting features. Cosmic strings, which is one feature of gravitational collapse in cylindrical symmetry are of particular interest since they are considered as possible "seeds" for galaxy formation and gravitational lenses. Furthermore, the ease with which naked singularities are formed in cylindrical symmetry needs further attention due to the apparent failure of cosmic censorship. Unlike the spherical gravitational collapse, collapse of cylindrical systems are not well understood. From this point of view, studying solutions with cylindrical symmetry is a worthy cause. Here, we present a new class of rotating charged cylindrical solutions. These are obtained by embedding the EinsteinMaxwell-dilaton solutions n $2+1$ dimensions in $3+1$ dimensions.

Now, we shall describe how the above rotating charged solutions in $2+1$ dimensions can be embedded in $3+1$ dimensions to obtain cylindrical solutions. Suppose, the new charged-dilaton solutions in $2+1$ dimensions are given by,

$$
d s^{2}=\tilde{h}_{i j} d x^{i} d x^{j}+e^{\chi} P(r)^{-2} d r^{2}
$$

as represented in eq.(39), with a dilaton field $\phi$, then, the four dimensional solution is given by,

$$
d s^{2}=\left(\tilde{h}_{i j} d x^{i} d x^{j}+e^{\chi} P(r)^{-2} d r^{2}\right) e^{4 \phi}+d z^{2} e^{-4 \phi}
$$

The corresponding potential $A_{\mu}$ will be the same as for $2+1$ dimensions. Embedding the electric solution in $2+1$ dimensions in eq.(49), one can obtain a new rotating charged solution in $3+1$ dimensions as,

$$
\begin{aligned}
d s_{3+1}^{2}= & \left(\frac{r^{2} L^{4} \alpha^{2} \gamma^{2}-\alpha^{2}}{L^{2}}\right) d t^{2}+2\left(\frac{r^{2} L^{4} \alpha \gamma\left(\alpha^{-1}+\alpha \beta \gamma\right)-\alpha^{2} \beta}{L^{2}}\right) d t d \varphi+ \\
& \left(\frac{r^{2} L^{4}\left(\alpha^{-1}+\alpha \beta \gamma\right)^{2}-\alpha^{2} \beta^{2}}{L^{2}}\right) d \varphi^{2}+r^{2 m^{2}} L^{2}\left(d r^{2}+d z^{2}\right)
\end{aligned}
$$

The electromagnetic potential is given by,

$$
\tilde{A}_{i} d x^{i}=\frac{\alpha Q}{2 c_{1} m} \frac{r^{-m}}{\left(c_{2} r^{-m}+c_{1} r^{m}\right)}(d t+\beta d \varphi)
$$

Here, $L$ is a function of $r$ given by,

$$
L=c_{1} r^{m}+c_{2} r^{-m}
$$


Embedding the magnetic solution in $2+1$ dimensions in eq.(52), one can obtain a new rotating charged solution in $3+1$ dimensions as,

$$
\begin{aligned}
& d s_{3+1}^{2}=\left(\frac{-r^{2+2 m^{2}} L^{4} \alpha^{2}+r^{2} \alpha^{2} \gamma^{2}}{r^{2} L^{2}}\right) d t^{2}+2\left(\frac{-r^{2+2 m^{2}} L^{4} \alpha^{2} \beta+r^{2} \alpha \gamma\left(\alpha^{-1}+\alpha \beta \gamma\right)}{r^{2} L^{2}}\right) d t d \varphi \\
& +\left(\frac{-r^{2+2 m^{2}} L^{4} \alpha^{2} \beta^{2}+r^{2}\left(\alpha^{-1}+\alpha \beta \gamma\right)^{2}}{r^{2} L^{2}}\right) d \varphi^{2}+r^{2} L^{2 m^{2}} d r^{2}+r^{2} L^{2} d z^{2}
\end{aligned}
$$

The electromagnetic potential is given by,

$$
\tilde{A}_{i} d x^{i}=\frac{-\alpha \gamma Q}{2 c_{1} m} \frac{r^{-m}}{\left(c_{2} r^{-m}+c_{1} r^{m}\right)}\left(d t+\left(\alpha^{-2} \gamma^{-1}+\beta\right) d \varphi\right)
$$

Here, $L$ is a function of $r$ given by,

$$
L=c_{1} r^{m}+c_{2} r^{-m}
$$

\section{Conclusions}

We have discussed a new family of charged-dilaton solutions in $2+1$ dimensions and charged solutions with cylindrical symmetry in $3+1$ dimensions. A class of static charged solutions in $2+1$ dimensions are obtained by compactifying solutions with cylindrical symmetry in $3+1$ dimensions. These space-times contain flat metric and naked singularities for certain values of the parameters considered. We have applied "T-duality" transformations to generate new rotating charged solutions in $2+1$ dimensions. These rotating charged solutions lead to new rotating charged solutions with cylindrical symmetry once embedded in $3+1$ dimensions.

Since all the solutions presented here contain wide number of parameters which may lead to space-times with differing physical properties, it is of a worthy cause to analysis these solutions in detail. A particular interesting solution to study further is the solution obtained from the Melvin solution in $3+1$ dimensions. It is well known that the Melvin solution has direct generalizations to low-energy string theory [17]. It has been shown that Melvin solution can be realized as the Kaluza-Klein reduction from flat higher dimensional space-time with non trivial identifications[18]. Therefore, the dimensionally reduced Melvin solution to $2+1$ dimensions has direct relations to higher dimensional space-times such as solutions of 11 dimensional Mtheory. Studying rotating Melvin solution would be an interesting exercise to further our understanding of higher dimensional physics.

It would be also interesting to embed the solutions discussed here in a supergravity theory arising from a low energy string theory along the lines of supersymmetric solutions to three dimensional heterotic string action considered by Bakas et.al.[19]. We hope to address these issues in the future. 


\section{References}

[1] K. Shiraishi, J. of Math. Phys. 34, 1480 (1993).

[2] D. Park and J.K. Kim, J. Math. Phys. 38, 2616 (1997).

[3] M. Bañados, C. Teitelboim and J. Zanelli, Phys. Rev. Lett. 69, 1849 (1992); M. Bañados, M. Henneaux, C. Teitelboim and J. Zanelli, Phys. Rev. D 48, 1506 (1993).

[4] K.C.K. Chan and R.B. Mann , Phys. Rev. D50, 6385 (1994).

[5] K.C.K. Chan and R.B. Mann, Phys. Lett. B371, 199 (1996).

[6] S. Fernando, Phys. Lett. B468 , 201 (1999).

[7] P.M. Sá, A. Kleber and J.P.S. Lemos, Class. Quan. Grav. 13, 199 (1996).

[8] T. Koikawa, T. Maki and A. Nakamula, Phys. Lett. B414, 45 (1997).

[9] S. Fernando, Gen. Rela. \& Grav. 34, 461 ( 2002)

[10] D. Kramer, H. Stephani, E. Herlt, M. MacCallum and E. Schmutzer, Exact Solutions of Einstein's Field Equations, ( Cambridge U.P., Cambridge, England, 1980).

[11] B. C. Muckherji, Bull. Calc. Math. Soc. 30, 95 (1938).

[12] M. A. Melvin, Phys. Lett. 8, 65 (1964).

[13] S. Deser, R. Jackiw and G. 'T Hooft, Ann. Phys. 152, 220 (1984).

[14] J.R. Gott and M. Alpert, Gen. Rela. \& Grav. 16, 243 (1984).

[15] Chiang-Mei Chen, Nucl. Phys. B544, 775 (1999).

[16] E. Bergoshoeff, B. Janssen and T. Ortin, Class. Quant. Grav. 13, 321 (1996).

[17] A.A. Tseytlin, Phys.Lett. B346, 55 (1995).

[18] F. Dowker, J.P. Gauntlett, S.B. Giddings and G.T. Horowitz, Phys. Rev. D50, 2662 (1994).

[19] I. Bakas, M. Bourdeau, and G. L. Cardoso, Nucl. Phys. B510, 103 (1998). 